\title{
Assessment of Arterial Stiffness, Brachial Haemodynamics, and Central Haemodynamics in Diabetic Hypertensives: A Pulse Wave Analysis-Based Case-Control Study from an Urban Area of West India
}

\author{
Jayesh Dalpatbhai Solanki ${ }^{a}$ Sunil J. Panjwani ${ }^{b}$ Ravi Kanubhai Patel ${ }^{c}$ \\ Devanshi Nishantbhai Bhatt ${ }^{c}$ Param Jagdeep Kakadia ${ }^{c}$ Chinmay J. Shah ${ }^{a}$ \\ aDepartment of Physiology, Government Medical College, Bhavnagar, Bhavnagar, India; ${ }^{\mathrm{b}}$ Department of General \\ Medicine, Government Medical College, Bhavnagar, Sir T General Hospital, Bhavnagar, India; ' Undergraduate MBBS \\ Student Working Under Physiology Department, Government Medical College, Bhavnagar, Bhavnagar, India
}

\section{Keywords}

Arterial stiffness - Blood pressure - Diabetic hypertensive · Haemodynamic Pulse wave analysis

\begin{abstract}
Introduction: Hypertension (HTN) and diabetes frequently coexist, imposing significant cardiovascular risk that is normally studied in terms of brachial blood pressure (bBP). Direct and superior parameters like central haemodynamics and arterial stiffness are studied scarcely. Pulse wave analysis (PWA) offers a non-invasive measurement of the same that we studied in diabetic hypertensives. Materials and Methods: We conducted a case-control study on 333 treated diabetic hypertensive cases and 333 euglycaemic normotensive controls. Oscillometric PWA was performed by Mobil-oGraph (IEM, Aachen, Germany). Parameters were further analysed in relation to gender, physical activity, body mass index (BMI), glycaemic control, blood pressure control, and
\end{abstract}

disease duration (cut-off 5 years). Multiple linear regressions were done to find significant associations. Results: Cases had significantly higher brachial haemodynamics (blood pressure, heart rate (HR), and rate pressure product); arterial stiffness measures (augmentation pressure, augmentation index, pulse wave velocity, total arterial stiffness, and pulse pressure amplification), and central haemodynamics (central blood pressure, cardiac output, stroke work) than controls. In the case group, female gender, BMI $\geq 23$, and uncontrolled blood pressures were significant factors that affected the results while other factors such as glycaemic control, physical activity, and duration did not. HR was significantly associated with study parameters. Brachial pressures were not significantly associated with corresponding aortic pressures. Conclusion: Diabetic hypertensives had adverse profile of cardiovascular parameters beyond bBP, related to female gender, and HTN and its control, more than that of diabetes. This baseline work suggests further study on these potential parameters.

(c) 2021 S. Karger AG, Base karger@karger.com www.karger.com/pls
(C) 2021 S. Karger AG, Basel

Karger ${ }^{\prime}=$
Correspondence to:

Jayesh Dalpatbhai Solanki,drjaymin_83@yahoo.com 


\section{Introduction}

Diabetes mellitus (DM) is a risk factor for hypertension (HTN) [1] and both coexist frequently. As compared to euglycaemic hypertensives, diabetic hypertensives have an added risk factor of hyperglycaemia that has additive adverse effect on cardiovascular health. Normotensive diabetics, on the other hand, do not receive antihypertensives that are suggested to prevent cardiovascular derangements [2-4]. But in the presence of DM with HTN, treatments are offered to correct both abnormal blood pressure and plasma glucose. This makes diabetic hypertensive patients an interesting population to study, especially with reference to cardiovascular health. Cardiovascular health is usually studied in terms of brachial blood pressure (bBP), but it has its limitations and subjectivity issue. Aortic stiffness, aortic blood pressure, and central haemodynamics are direct parameters inferring about the same. Central blood pressure (CBP) is superior to bBP and similarly central haemodynamics like cardiac output (CO) adds extra domain in the assessment of cardiovascular health. Arterial stiffness parameters like aortic pulse wave velocity, augmentation index are more stable parameters than bBP, indicating cumulative effects of various factors on cardiovascular ageing. The pulse wave analysis (PWA) method provides an estimate of the cardiovascular parameters, such as CBP, central haemodynamics, and arterial stiffness [5, 6]. Mobil-o-Graph is one of those validated, calibrated devices based on oscillometric PWA that allows objective measurement of these parameters with proven reproducibility. We have reported Mobil-o-graph-based PWA studies on euglycaemic hypertensives [7] and normotensive type 1 [8] and type 2 [9] diabetics of our population. With DM or HTN alone, we found adverse profile of PWA parameters of cardiovascular ageing, mostly independent of conventional risk factors like blood pressure, disease duration, and anthropometric parameters. But how these parameters are affected, if both DM and HTN coexist, is unknown in our population, more so, in light of their frequent coexistence and with poor control of either disease in most. We set out to study arterial stiffness, and brachial and central haemodynamics in diabetic hypertensives using the same PWA device and protocol for a case-control study.

\section{Materials and Methods}

Study Design and Participants

The study protocol was approved by the institutional review board of our college (HEC No. 678/2015 dated March 31, 2015).
Physiology and Medicine departments conducted a case-control study from 18 June 2015 to 20 May 2018. We studied ambulatory patients of the Department of Medicine and General Outdoor Patient department attending a tertiary care teaching government hospital, affiliated to a government medical college.

\section{Inclusion and Exclusion Criteria}

We included known, under-treatment hypertensive diabetics, taking regular antihypertensives and antidiabetics since at least 6 months, aged $\leq 65$ years, of either sex, non-smoking, non-alcoholic, not known to have any acute or chronic systemic disease, ready for written informed consent. Apart from non-compliance with these criteria, we excluded subjects using any alternative system of medicines/life style managements like yoga and meditation.

\section{Study Groups}

The sample size was calculated by Raosoft software (free online software; Raosoft, Inc., Seattle, WA, USA). To have 95\% confidence level, 5\% precision, and considering response distribution of $30 \%$, a sample size of 323 was minimum required for a city having a population of 6 lakh.

We screened and enrolled 791 hypertensives from the Medicine Outdoor Patient Department by simple random sampling. We excluded 149 new hypertensives (duration $<6$ months) and 263 euglycaemic hypertensives, and the remaining 379 participants with concomitant diabetes were considered for the current study. Of them, we excluded 25 with a history of irregular treatment, 10 with use of life style modification, 3 owing to irregular pulse wave recording, 4 with morbid obesity, and 4 due to arm circumference beyond available cuff size. So, the case group finally had 333 diabetic hypertensives.

For comparison as controls, we selected 333 apparently healthy, euglycaemic, normotensive participants from the available pool of 1,226 healthy controls $[5,6]$ used in previous normative studies. Controls were matched head to head to cases for gender; and for age and body mass index (BMI) to nearest possible.

\section{Participant Assessment and Definitions}

All participants were personally interviewed in the form of questionnaires regarding general and demographic characteristics (age, height, weight, and BMI), risk factors, self-reported moderate physical activity (defined as regular moderate exercise for at least $30 \mathrm{~min}$ for minimum 5 days a week), and relevant disease history. Detailed history of pharmacotherapy used was elicited from each case and regularity was confirmed by the patients' case report chart. Systolic blood pressure $(\mathrm{SBP}) \geq 140 \mathrm{~mm} \mathrm{Hg}$ and diastolic blood pressure (DBP) $\geq 90 \mathrm{~mm} \mathrm{Hg}$ or use of antihypertensive medication was defined as HTN. Brachial SBP $<140 \mathrm{~mm} \mathrm{Hg}$ and DBP $<90 \mathrm{~mm} \mathrm{Hg}$ were taken as blood pressure control (JNC 8 criteria for the management of HTN in adult). Glycaemic control was defined as per American Diabetes Association guidelines 2014 based on fasting plasma glucose $(<130 \mathrm{mg} / \mathrm{dL})$ and 2 -h plasma glucose $(2 \mathrm{hPG}<180 \mathrm{mg} / \mathrm{dL})$.

\section{Instrument Used}

We used a portable, personal computer attached, calibrated instrument Mobil-o-Graph (IEM GMBH, Stolberg, Germany) that has been validated against direct invasive measurement and has reproducibility comparable to cardiac magnetic resonance [10, 11]. It undergoes oscillometric pressure PWA as per protocol designed by the European Society of Hypertension. 
Table 1. Comparison of baseline and study parameters between cases and matched controls; and male cases versus female cases

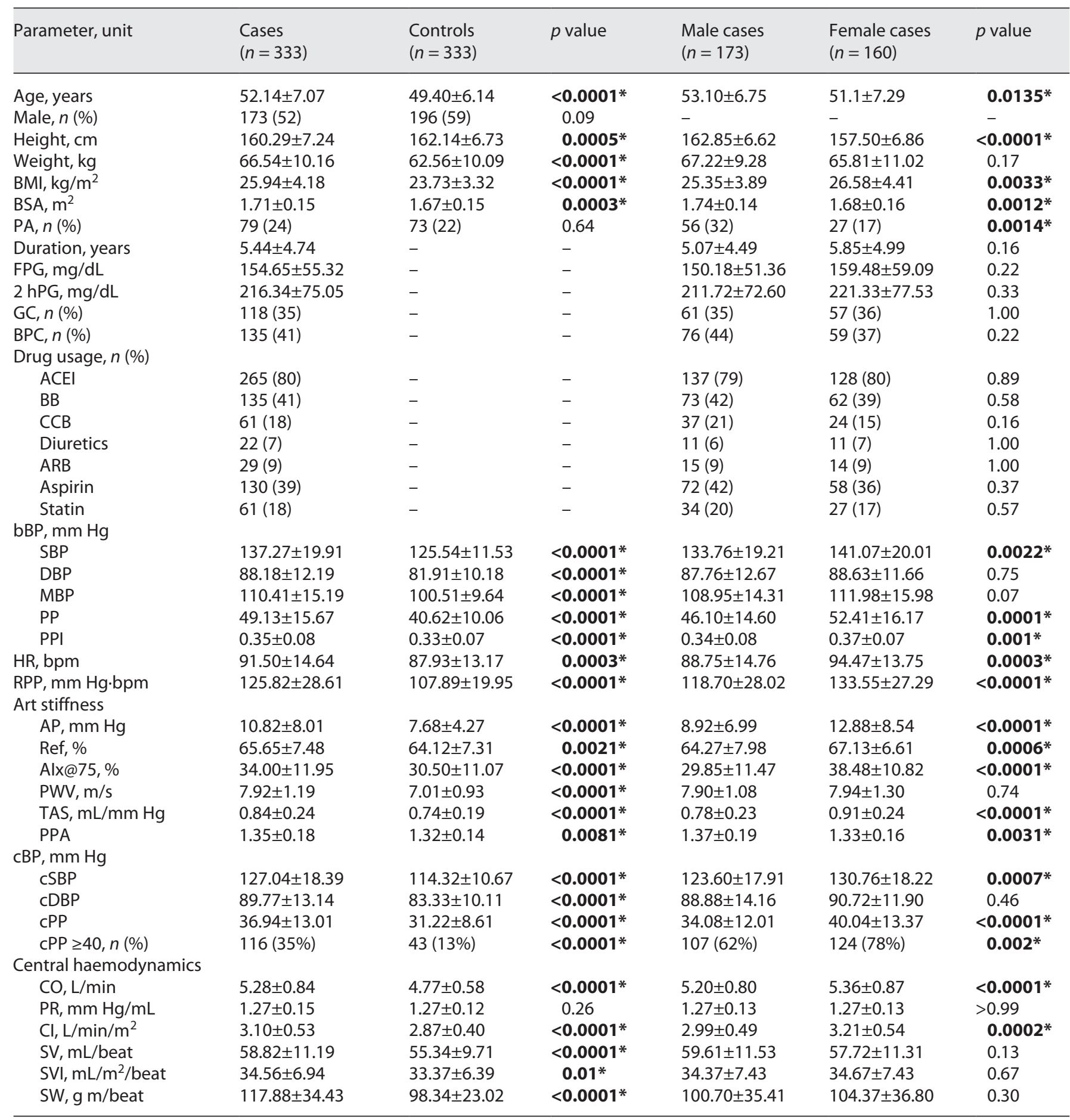

CCB, calcium channel blocker; ACEl, Angiotensin-converting enzyme inhibitor; ARB, Angiotensin II receptor blocker; BMI, body mass index; PA, physical activity; FPG, fasting plasma glucose; 2 hPG, 2 h plasma glucose; GC, glycaemic control; BPC, blood pressure control; BB, $\beta$-blocker; bBP, brachial blood pressure; SBP, systolic blood pressure; DBP, diastolic blood pressure; MBP, mean blood pressure; PP, pulse pressure; PPI, pulse pressure index; HR, heart rate; RPP, rate pressure product; AP, augmentation pressure; Ref, reflection percentage; Alx@75, augmentation index at heart rate 75 beats per minute; PWV, pulse wave velocity; TAS, total arterial stiffness; PPA, pulse pressure amplification; cSBP, central systolic blood pressure; CDBP, central diastolic blood pressure; cPP, central pulse pressure; CO, cardiac output; PR, peripheral resistance; Cl, cardiac index; SV, stroke volume; SVI, stroke volume index; SW, stroke work. * Indicates statistical significance.

Pulse Wave Analysis in Diabetic Hypertensives
Pulse 2021;9:89-98

DOI: $10.1159 / 000519357$ 
Table 2. Comparison of baseline and study parameters between subgroups of cases based on physical activity (present or absent) and BMI (cut-off 23)

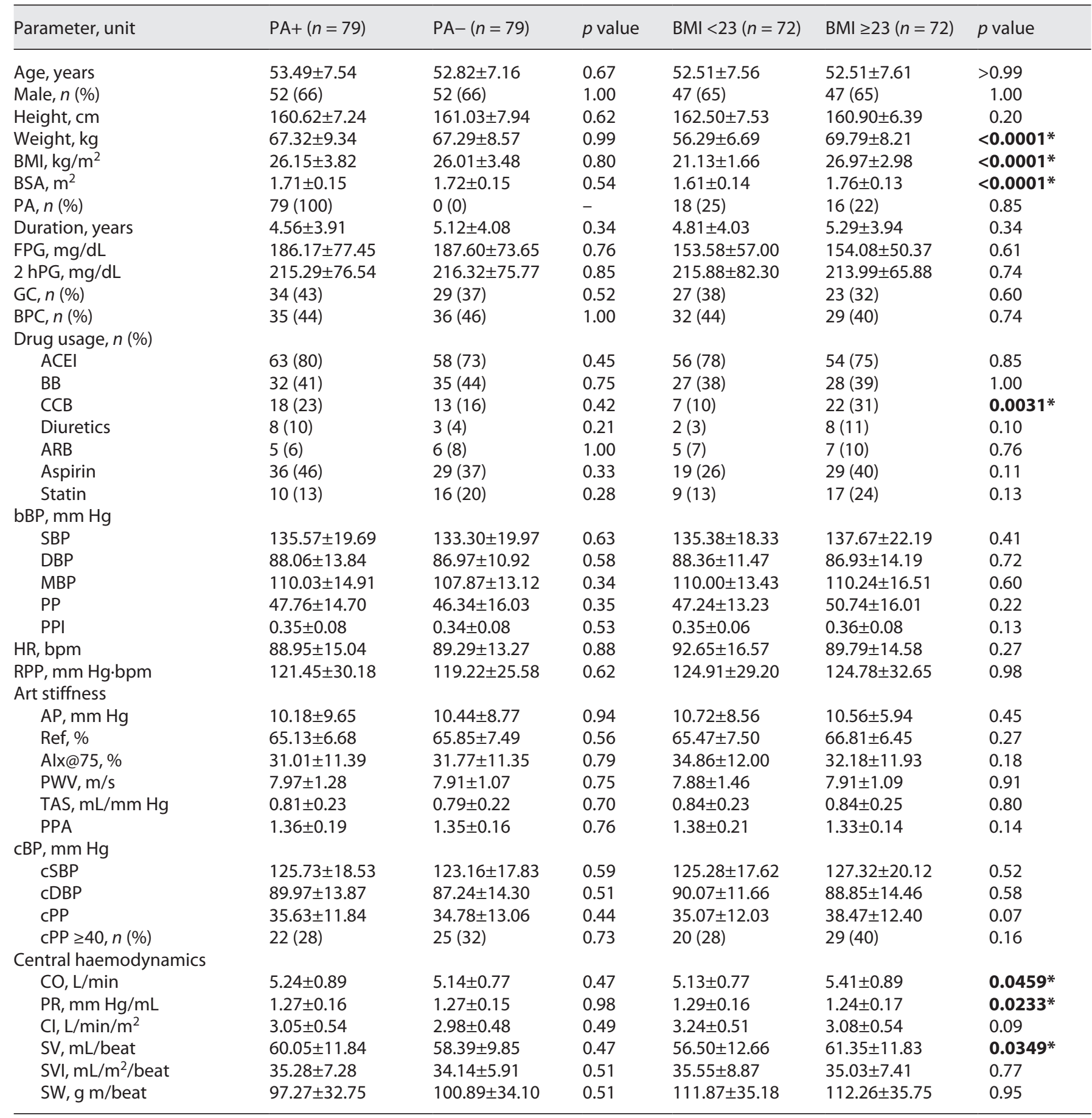

CCB, calcium channel blocker; ACEl, Angiotensin-converting enzyme inhibitor; ARB, Angiotensin II receptor blocker; BMI, body mass index; PA, physical activity; FPG, fasting plasma glucose; 2 hPG, 2 h plasma glucose; GC, glycaemic control; BPC, blood pressure control; BB, $\beta$-blocker; bBP, brachial blood pressure; SBP, systolic blood pressure; DBP, diastolic blood pressure; MBP, mean blood pressure; PP, pulse pressure; PPI, pulse pressure index; HR, heart rate; RPP, rate pressure product; AP, augmentation pressure; Ref, reflection percentage; Alx@75, augmentation index at heart rate 75 beats per minute; PWV, pulse wave velocity; TAS, total arterial stiffness; PPA, pulse pressure amplification; CSBP, central systolic blood pressure; CDBP, central diastolic blood pressure; cPP, central pulse pressure; CO, cardiac output; $\mathrm{PR}$, peripheral resistance; Cl, cardiac index; SV, stroke volume; SVI, stroke volume index; SW, stroke work. * Indicates statistical significance.

92

Pulse 2021;9:89-98

DOI: $10.1159 / 000519357$
Solanki/Panjwani/Patel/Bhatt/Kakadia/ Shah 
Pressure oscillations from brachial arterial pulsation are transmitted to bBP cuff and measured by a transducer to be fed into a microprocessor. Computerized software records pulse wave of brachial artery and by validated generalized transfer factor derives central aortic pulse wave. It further undergoes point-based and area-based analysis by computer to derive various cardiovascular parameters.

\section{Measurement Protocol [10]}

A blood pressure cuff of appropriate size (mid-arm circumference: $20-24 \mathrm{~cm}=$ small size, $24-32 \mathrm{~cm}=$ medium size, $32-38 \mathrm{~cm}=$ large size) was chosen and applied to the left arm using standard protocol. All readings were taken after $10 \mathrm{~min}$ of rest, in a calm room without external influences and avoiding arm movement, in post-absorptive phase while the participant avoided smoking or alcohol for $12 \mathrm{~h}$ before measurement. Only very good data quality records were considered as reading. Each reading averaged parameters based on multiple recorded pulse wave over $10 \mathrm{~s}$ and the average of such 2 readings was taken for each participant as final observation.

Parameters measured (as described in the previous study $[5,6]$ ).

1. Heart rate (HR), BMI, body surface area (BSA).

2. bBP-systolic, diastolic, pulse (bPP), mean (bMBP).

3. cBP-systolic, diastolic, pulse.

4. Central haemodynamics-CO, cardiac index, peripheral resistance ( $\mathrm{PR}=$ derived by software from MBP and $\mathrm{CO}$ ).

5. Arterial stiffness-augmentation pressure, augmentation index at HR 75 per minute (AIx@75), reflection magnitude percentage (Ref \%), aortic pulse wave velocity.

Parameters derived (as described in the previous study $[5,6]$ ).

1. Rate pressure product $(\mathrm{RPP})-(\mathrm{HR}$ per minute $) \times(\mathrm{SBP}) \times 10^{-2}$.

2. Stroke volume $(\mathrm{SV})-\mathrm{CO} / \mathrm{HR}$.

3. SV index $-\mathrm{SV} /$ body surface area.

4. Stroke work - (pulse pressure $) \times(\mathrm{SV}) \times 0.0144$.

5. Total arterial stiffness - pulse pressure/SV.

6. Pulse pressure index (PPI) - pulse pressure/SBP

7. Pulse pressure amplification (PPA) - brachial pulse pressure/ aortic pulse pressure.

\section{Statistical Analysis}

The data were entered into and sorted by an Excel spreadsheet. Numerical data were expressed as mean \pm standard deviation until indicated specifically, and qualitative data were expressed as number (percentage). Statistical calculations were done by GraphPad InStat 3 software (demo version free software of GraphPad Software, Inc., San Diego, CA, USA). Numerical data were compared for difference in mean/median distribution using unpaired $t$ test or Mann-Whitney test, depending on outcome of Normality test. We compared the difference in distribution of qualitative data by Normality test or $\chi^{2}$ test. Taking age, anthropometric parameters, brachial haemodynamics, duration of disease, and glycaemic control values as independent parameters and central haemodynamics and arterial stiffness as dependant parameters, multiple linear regression tests were applied. Statistical significance level was set at $p$ value $<0.05$.

\section{Results}

Cases $(n=333)$ and controls $(n=333)$ had comparable gender distribution and physical activity status. Cases (mean disease duration 5.44 years) were significantly shorter, older, and obese than controls. Glycaemic or pressure control was present in two-fifth of cases. Angiotensin-converting enzyme inhibitor was the commonest antihypertensive drug followed by $\beta$-blocker. Most study parameters including bBPs, RPP, vascular stiffness, and central haemodynamic parameters were higher in cases than controls. Statistical significance was present for all except PR (Table 1). In the case group, we compared males $(n=173)$ and females $(n=160)$. These subgroups were comparable for weight, duration, blood pressure control, glycaemic control, and use of pharmacotherapy. In comparison, females were significantly shorter and obese while males were significantly older and more physically active. Parameters of brachial haemodynamics (except DBP and MBP), arterial stiffness (except PWV), and central haemodynamics (only CO and cardiac index) were significantly higher in females than males (Table 1 ).

Profile of PWA parameters was not significantly different in physically active cases than matched and comparable physically inactive cases. As compared to cases with $\mathrm{BMI}<23$, cases with $\mathrm{BMI} \geq 23$ had raised PWA parameters but statistical significance was lacking for all parameters except $\mathrm{CO}$ (Table 2).

Cases with controlled blood pressure had significantly shorter height, lesser weight but higher percentage of $\beta$-blocker usage than cases with uncontrolled blood pressure. Cases with uncontrolled blood pressure had higher CBP, central haemodynamics, and arterial stiffness. Statistical significance was present for most observed differences except for HR, reflection magnitude, PPA, and PPI. Good glycaemics and poor glycaemics were comparable for all study parameters except higher disease duration, $\mathrm{HR}, \mathrm{RPP}, \mathrm{CO}$, and PR in the later group compared to the former group (Table 3 ).

New cases were not significantly different from matched old cases with respect to baseline and study parameters (except PPA and SV index). Cases with longer disease duration ( $>5$ years) had insignificantly different profile of study parameters as compared to cases with shorter disease duration ( $\leq 5$ years), except PPI, total arterial stiffness, and stroke work, which were significantly higher in the former group than in the later group (Table 4).

HR was significantly associated with arterial stiffness and central haemodynamic parameters. Age with PWV; bMBP with AIx and total arterial stiffness; weight with augmentation pressure, $\mathrm{cBP}$-systolic, central pulse pressure; and height with $\mathrm{PP}$ were significant associations revealed by multiple linear regressions in the case group. Most bBP parameters were not significantly associated with corresponding CBPs parameters (Table 5). 
Table 3. Comparison of baseline and study parameters between subgroups of cases based on blood pressure control (present or absent) and glycaemic control (present or absent)

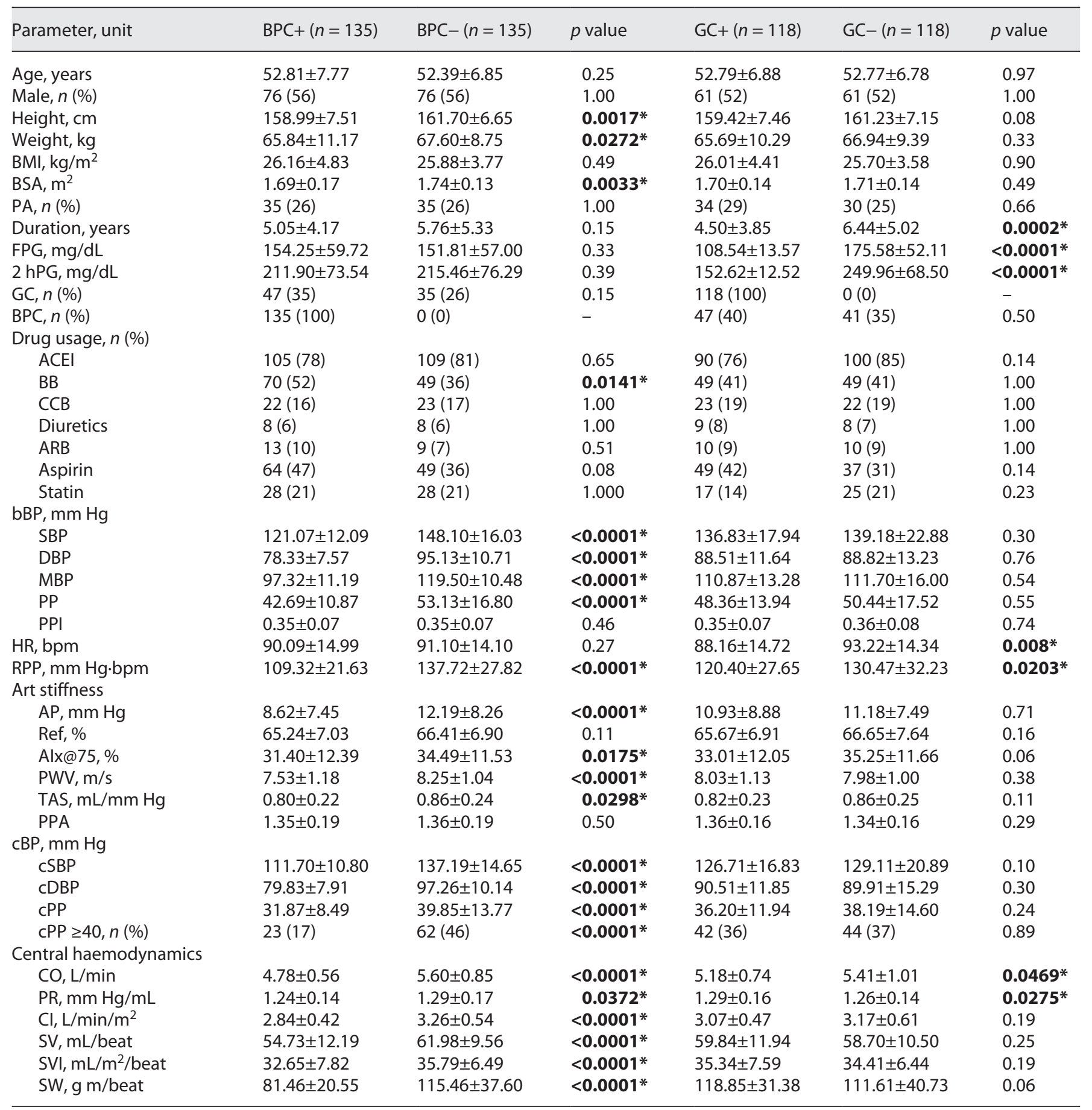

CCB, calcium channel blocker; ACEl, Angiotensin-converting enzyme inhibitor; ARB, Angiotensin II receptor blocker; BMI, body mass index; PA, physical activity; FPG, fasting plasma glucose; 2 hPG, 2 h plasma glucose; GC, glycaemic control; BPC, blood pressure control; BB, $\beta$-blocker; bBP, brachial blood pressure; SBP, systolic blood pressure; DBP, diastolic blood pressure; MBP, mean blood pressure; PP, pulse pressure; PPI, pulse pressure index; HR, heart rate; RPP, rate pressure product; AP, augmentation pressure; Ref, reflection percentage; Alx@75, augmentation index at heart rate 75 beats per minute; PWV, pulse wave velocity; TAS, total arterial stiffness; PPA, pulse pressure amplification; CSBP, central systolic blood pressure; CDBP, central diastolic blood pressure; cPP, central pulse pressure; CO, cardiac output; PR, peripheral resistance; Cl, cardiac index; SV, stroke volume; SVI, stroke volume index; SW, stroke work. * Indicates statistical significance.

94

Pulse 2021;9:89-98

DOI: $10.1159 / 000519357$
Solanki/Panjwani/Patel/Bhatt/Kakadia/ Shah 
Table 4. Comparison of baseline and study parameters between subgroups of cases based on disease duration (new vs. old; duration more than vs. less than 5 years)

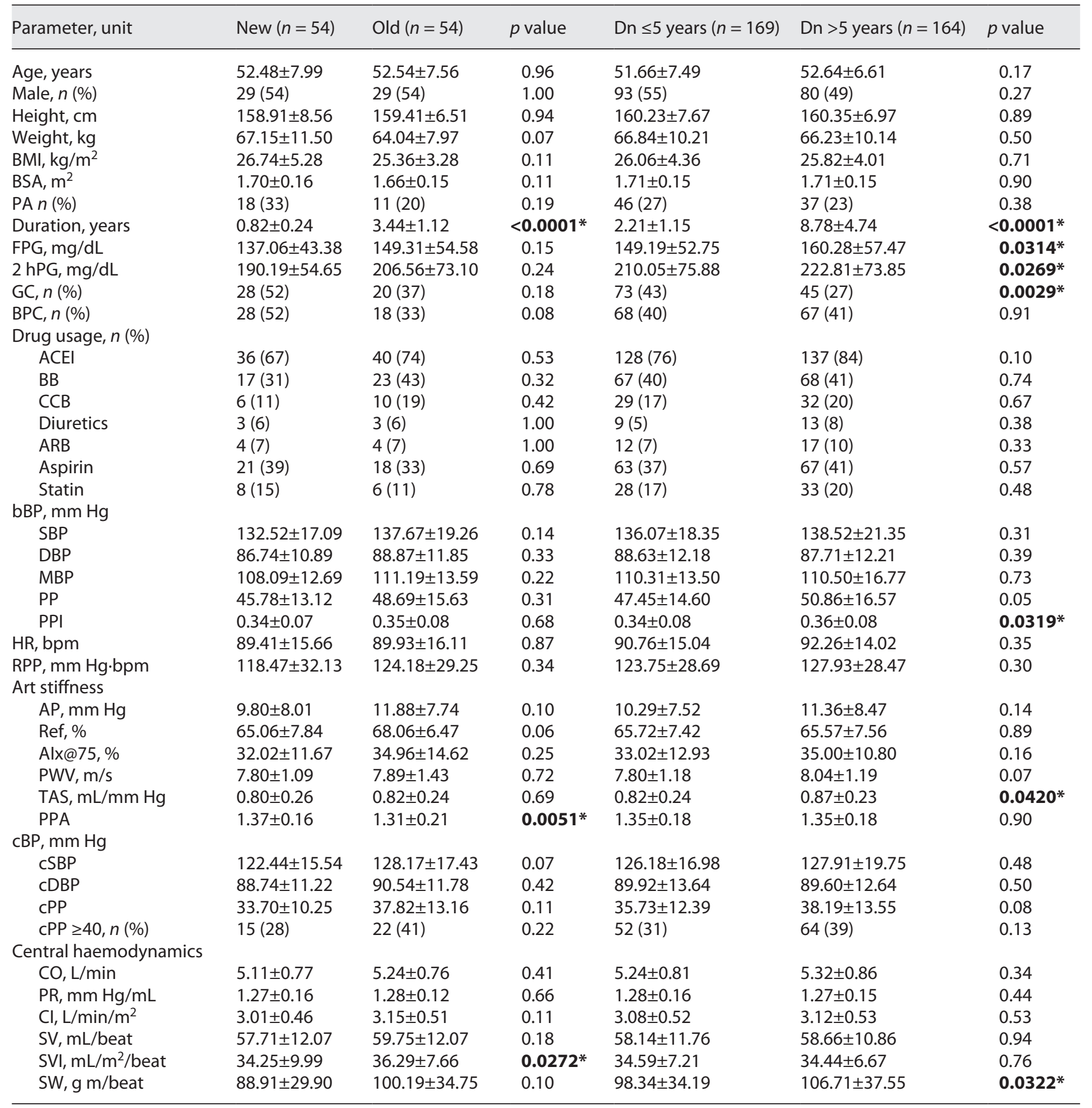

CCB, calcium channel blocker; ACEl, Angiotensin-converting enzyme inhibitor; ARB, Angiotensin II receptor blocker; BMI, body mass index; PA, physical activity; FPG, fasting plasma glucose; 2 hPG, 2 h plasma glucose; GC, glycaemic control; BPC, blood pressure control; BB, $\beta$-blocker; bBP, brachial blood pressure; SBP, systolic blood pressure; DBP, diastolic blood pressure; MBP, mean blood pressure; PP, pulse pressure; PPI, pulse pressure index; HR, heart rate; RPP, rate pressure product; AP, augmentation pressure; Ref, reflection percentage; Alx@75, augmentation index at heart rate 75 beats per minute; PWV, pulse wave velocity; TAS, total arterial stiffness; PPA, pulse pressure amplification; CSBP, central systolic blood pressure; CDBP, central diastolic blood pressure; cPP, central pulse pressure; CO, cardiac output; PR, peripheral resistance; Cl, cardiac index; SV, stroke volume; SVI, stroke volume index; SW, stroke work. * Indicates statistical significance.

Pulse Wave Analysis in Diabetic Hypertensives
Pulse 2021;9:89-98

DOI: $10.1159 / 000519357$ 
Table 5. Calculation of predictors for dependant variables by multiple linear regressions ( $r_{\text {partial }}$ values) in case group

\begin{tabular}{|c|c|c|c|c|c|c|c|c|c|}
\hline Parameters & AP & Alx@75 & aPWV & TAS & CSBP & cDBP & CPP & $\mathrm{CO}$ & SW \\
\hline Age & 0.02 & -0.04 & $0.77 * *$ & -0.02 & -0.05 & -0.04 & -0.03 & 0.02 & 0.01 \\
\hline Height & 0.10 & 0.02 & 0.02 & 0.05 & 0.10 & -0.04 & $0.13^{*}$ & -0.06 & 0.02 \\
\hline Weight & $-0.12 *$ & -0.07 & -0.04 & -0.09 & $-0.12 *$ & 0.02 & $-0.15^{*}$ & 0.09 & 0.02 \\
\hline BMI & -0.10 & 0.04 & 0.03 & 0.07 & $0.12^{*}$ & -0.02 & $0.15^{*}$ & -0.07 & -0.00 \\
\hline SBP & 0.02 & -0.08 & 0.05 & -0.05 & $0.15^{*}$ & 0.06 & 0.06 & 0.03 & 0.09 \\
\hline DBP & -0.03 & 0.06 & -0.02 & -0.03 & 0.07 & 0.08 & -0.04 & 0.04 & 0.04 \\
\hline MBP & 0.08 & $0.14 *$ & 0.01 & $0.11 *$ & -0.07 & 0.02 & -0.09 & -0.10 & -0.08 \\
\hline $\mathrm{HR}$ & $-0.16 *$ & $0.58 * *$ & $0.11 *$ & $0.81 * *$ & $-0.13^{*}$ & -0.02 & $-0.18^{*}$ & $0.32 * *$ & $-0.78 * *$ \\
\hline PP & 0.02 & 0.09 & 0.00 & $0.17^{*}$ & 0.02 & -0.06 & 0.10 & 0.04 & 0.04 \\
\hline Duration & 0.03 & 0.06 & -0.00 & -0.04 & 0.04 & 0.05 & 0.02 & 0.02 & 0.02 \\
\hline FBS & -0.02 & -0.02 & 0.01 & 0.01 & -0.04 & 0.06 & -0.06 & -0.01 & -0.01 \\
\hline PP2BS & 0.09 & 0.03 & 0.00 & 0.01 & 0.04 & -0.08 & 0.09 & -0.02 & -0.01 \\
\hline
\end{tabular}

$C C B$, calcium channel blocker; ACEl, Angiotensin-converting enzyme inhibitor; ARB, Angiotensin II receptor blocker; BMI, body mass index; PA, physical activity; FPG, fasting plasma glucose; 2 hPG, 2 h plasma glucose; GC, glycaemic control; BPC, blood pressure control; BB, $\beta$-blocker; bBP, brachial blood pressure; $S B P$, systolic blood pressure; DBP, diastolic blood pressure; MBP, mean blood pressure; PP, pulse pressure; PPI, pulse pressure index; HR, heart rate; RPP, rate pressure product; AP, augmentation pressure; Ref, reflection percentage; Alx@75, augmentation index at heart rate 75 beats per minute; PWV, pulse wave velocity; TAS, total arterial stiffness; PPA, pulse pressure amplification; cSBP, central systolic blood pressure; CDBP, central diastolic blood pressure; $C P P$, central pulse pressure; $\mathrm{CO}$, cardiac output; $\mathrm{PR}$, peripheral resistance; $\mathrm{Cl}$, cardiac index; SV, stroke volume; SVI, stroke volume index; SW, stroke work; aPWV, aortic pulse wave velocity. Significant results are highlighted in bold. ${ }^{*} p<0.05 .{ }^{* *} p<0.0001$.

\section{Discussion}

The present study is by far the first Mobil-o-graph-based study in middle-aged, urban, Indian diabetic hypertensives. Diabetic hypertensives had higher brachial haemodynamics, central haemodynamics, and arterial stiffness than controls despite antihypertensive and antidiabetic therapy. These are in line with previous studies of PWA done in euglycaemic hypertensives [7] and normotensive diabetics [9] of our region. However, cases were significantly older and obese, so exact matching with controls was not possible. It can also be explained by poor glycaemic and blood pressure control despite therapy, which is one of the features of Indian diabetics, hypertensives $[12,13]$ and indicates the felt need for strict control to have better cardiovascular health. Apart from dual threat of HTN and diabetes [14], it can also be due to higher prevalence of physical inactivity, ethnic predisposition, delayed diagnosis, and lack of life style modification and underlying cardiac disease as suggested by use of $\beta$-blockers in $41 \%$ cases. Insulin resistance, ageing, arterial stiffness, HTN/diabetes, and cardiovascular risk are all linked to each other [15] and the same is present even in treated individuals. Parameters like PPI, PPA, and PR were not significantly different in cases than in controls alike previous $[9,11]$ studies. These parameters are from peripheral arteries which are better controlled by antihypertensive therapy and reflected as controlled bBP. This accelerated cardiovascular profile indicates increased cardiac workload that can produce adverse effect on heart itself as well as on other target organs like brain and kidney $[12,14,16]$.

Females had significantly higher PWA parameters than males, in line with previous studies [7,9]. Mean age 51 years [17], sex hormone-specific differences, shorter height, physical inactivity, and higher mean BMI can explain female disadvantages. Higher stiffness and accelerated haemodynamics can explain higher cardiovascular risk in middle-aged females than males. Self-reported moderate physical activity and BMI $<23$ had no significant impact on PWA results in diabetic hypertensive study participants. This is in accordance with the study on euglycaemic hypertensives [7] but in contrast to the study on normotensive diabetics [9] where impact was found significant. It suggests that obesity, physical activity, and weight management are significantly more important in diabetics without HTN, but with incident HTN their effects are overshadowed by other risk factors. It can also be due to the lack of intense exercising and that fact that physical activity was self-reported with possible subjective bias. It can be further studied by inclusion of qualitative body fat measurements that provide better insight [18] into relation between body adiposity and abnormal PWA profile. Despite lack of statistical significance here, physical exercise and optimum 
BMI management are good preventive measures for overall health and prognosis, more so in presence of diabetes [19].

Blood pressure control was advantageous for good cardiovascular PWA profile, in line with others [20] and our study [7] on euglycaemic hypertensives. Lowering of blood pressure by first-line antihypertensives is known to decrease cardiovascular risk without any class difference [13] and the same was found as better arterial stiffness and haemodynamic profile in cases with controlled blood pressure. Such benefit of early diagnosis and optimum blood pressure control must be offered to every hypertensive. Contrastingly to blood pressure control effect, lack of impact of glycaemic control was found in our study, but in line with the study [9] in normotensive type 2 diabetics. It can be explained by ethnicity risk, use of plasma glucose than $\mathrm{HbAlc}$, and poor glycaemic control (40\%) in most cases. Alike us, Gordin et al. [21] found that in patients with type 2 diabetes and albuminuria, hyperglycaemia induces an increase in stiffness of intermediate-sized arteries with no changes in other parts of the arterial bed (like aorta in our case). Similarly, Chang et al. [22] documented that short-term glycaemic control did not influence the arterial stiffness in patients with type $2 \mathrm{DM}$ and HTN. HR and rate pressure product were the only PWA parameters affected significantly and adversely by glycaemic control. This could be due to cardiac dysautonomia induced by diabetes, which is previously reported [23] in our diabetic population as higher prevalence of reduced HR variability. So, HTN and blood pressure had more impact on PWA cardiovascular parameters than diabetes and glycaemic control. We did not find significant difference between new or old cases and cases with duration less than or more than 5 years with respect to PWA parameters, alike previous studies [7, 9]. It indicates the importance of the presence of these diseases, their early diagnosis, and prompt treatment more than that of further chronicity of these incurable diseases.

Multiple linear regressions revealed results in diabetic hypertensives to be in line with diabetic [9] and hypertensive [7] population we reported. Except a few, most study parameters were not significantly associated with age, anthropometrics, and bBPs, pointing towards significance of these PWA parameters to complement routinely, subjectively measured bBP. Despite use of $\beta$-blockers in $41 \%$ cases, HR was comparatively high and association of HR with PWA parameters shows the potential of details that one can obtain by arterial pulse examination. Lack of association between aortic and bBP was in line with our previous studies indicating an add-on benefit of CBP in disease monitoring.

An adverse positive feedback cycle is present between type 2 diabetes and HTN that may explain the high coexis- tence of these conditions and that greatly increases cardiovascular disease risk [24]. As reported recently, the acceleration of cardiovascular ageing is suggested to start in young to middle-aged individuals in the absence of DM or HTN if there is a family history of these diseases is present. $[25,26]$. Our result in diabetic hypertensives highlights HTN as a factor that overshadows diabetes when both coexist. More work is needed to ascertain the role of these underrated parameters like arterial stiffness and central haemodynamics, with vertical follow-up and detailed biomarker testing. This, being a baseline study, asks for further interventional and vertical studies for reinforcement of our results. There is also a need to ascertain the role of other risk factors not studied due to the limitations of our study.

There were few limitations of our study like cross-sectional nature, moderate sample size, lack of baseline data or follow-up, absence of biochemical investigations, use of $\beta$-blocker in many participants that modifies PWA parameters, lack of adjustment for dietary pattern, socioeconomic status, and use of chewable tobacco.

\section{Conclusion}

Gujarati diabetic hypertensives showed accelerated profile of cardiovascular parameters beyond bBP; not associated with conventional confounders; related to female gender, blood pressure control; and not related to physical activity, BMI, glycaemic control, or disease duration. It suggests HTN as a major cause than diabetes for cardiovascular progeria when both coexist; hints towards early diagnosis and prompt control as a potential measure left to be offered and explored further.

\section{Acknowledgments}

We are thankful to the physicians, medical officers, and resident doctors of the Department of Medicine as well as faculties and staff of Physiology Department of Government Medical College, Bhavnagar, Bhavnagar, Gujarat, India, for their contribution and guidance in the study.

\section{Statement of Ethics}

The study was approved by the institutional ethical committee according to the Helsinki Declaration of the World Medical Association (2000). The study protocol was approved by the institutional review board of our college (HEC) No. 678/2015 dated March 31, 2015). All study participants provided signed informed consent prior to study initiation. 


\section{Conflict of Interest Statement}

The authors have no conflicts of interest to declare.

\section{Funding Sources}

This study did not receive any specific research grant from funding agencies in the public, commercial, or not-for-profit sectors.

\section{Author Contributions}

Jayesh D. Solanki contributed to all aspects of the study. Ravi Kanubhai Patel, Devanshi Nishantbhai Bhatt, and Param J. Kakadia contributed to data collections, data analysis, and statistical analysis. Sunil J. Panjwani and Chinmay J. Shah contributed to concept, design, and literature search. All authors contributed to manuscript editing, manuscript review and all are guarantors.

\section{Data Availability Statement}

Research data are not publicly available.

\section{References}

1 Singh S, Gupta NR, Raza ST, Kapoor A, Singh $\mathrm{P}$. Association of hypertension and its risk factor in type II diabetes mellitus patients. Asian J Med Sci. 2021;12(1):28-33.

2 Solanki JD, Basida SD, Mehta HB, Panjwani SJ, Gadhavi BP. Comparative study of cardiac autonomic status by heart rate variability between under-treatment normotensive and hypertensive known type 2 diabetics. Indian Heart J. 2017;69(1):52-6.

3 Solanki J, Patel K, Lalwani N, Mehta H, Shah C, Lakhtaria M. QT corrected for heart rate and qtc dispersion in Gujarati type 2 diabetics predominantly using preventive pharmacotherapy and with very low electrocardiogram left ventricular hypertrophy. J Diabetol. 2017; 8(3):86-91.

4 Solanki JD, Makwana AH, Mehta HB, Gokhale PA, Shah CJ. Hypertension in type 2 diabetes mellitus: effect of the disease and treatment on development of peripheral artery disease. Int J Diabetes Dev Ctries. 2015; 35(S3):380-4.

5 Solanki J, Mehta H, Shah C. Aortic blood pressure and central hemodynamics measured by noninvasive pulse wave analysis in Gujarati normotensives. Int J Clin Exp Physiol. 2018;5(2):75-80.

6 Solanki J, Mehta H, Shah C. Aortic pulse wave velocity and augmentation index@75 measured by oscillometric pulse wave analysis in Gujarati nonhypertensives. Vasc Invest Ther. 2018;1(2):50-5.

7 Solanki JD, Mehta HB, Panjwani SJ, Munshi HB, Shah CJ. Central hemodynamics and arterial stiffness by oscillometric pulse-wave analysis in treated Gujarati euglycemic hypertensives: a case-control study. J Family Med Prim Care. 2019;8:2047-54.

8 Solanki J, Munshi H. Pulse wave analysis in Gujarati type 1 diabetics: a case control study. Natl J Integr Res Med. 2018;9(6):59-65.

9 Solanki JD, Munshi HB, Mehta HB, Shah CJ. Central hemodynamics and arterial stiffness in Gujarati diabetics not receiving any antihypertensive: a case-control study based on oscillometric pulse wave analysis. J Family Med Prim Care. 2019;8:1352-8.
10 Weiss W, Gohlisch C, Harsch-Gladisch C, Tölle M, Zidek W, van der Giet M. Oscillometric estimation of central blood pressure: validation of the Mobil-O-Graph in comparison with the SphygmoCor device. Blood Press Monit. 2012;17(3):128-31.

11 Feistritzer HJ, Reinstadler SJ, Klug G, Kremser C, Seidner B, Esterhammer R, et al. Comparison of an oscillometric method with cardiac magnetic resonance for the analysis of aortic pulse wave velocity. PLoS One. 2015;10: e0116862.

12 Solanki JD, Patel RB, Hadiyel IN, Mehta HB, Munshi HB, Kakadiya PJ. Association of brachial and central hemodynamic parameters to eGFR and proteinuria in Gujarati diabetics with mild-to-moderate nephropathy. J Family Med Prim Care. 2019;8:2965-70.

13 Solanki J, Mehta H, Panjwani S, Munshi H, Shah C. Effect of antihypertensive pharmacotherapy on oscillometric pulse wave analysis parameters in treated Gujarati hypertensives: a cross-sectional study. J Pharmacol Pharmacother. 2018;9(3):153-9.

14 Ren Q, Ma C, Wang J, Guo X, Ji L; ATTEND Investigators. Albuminuria and other target organ damage in Chinese patients with hypertension and diabetes: a data analysis based on the ATTEND study. J Diabetess Complications. 2020;34(1):107470.

15 Tsimihodimos V, Gonzalez-Villalpando C, Meigs JB, Ferrannini E. Hypertension and diabetes mellitus: coprediction and time trajectories. Hypertension. 2018;71(3):422-8.

16 Solanki JD, Patel RB, Hadiyel IN, Mehta HB, Munshi HB, Kakadiya PJ. Association of brachial and central hemodynamic parameters to eGFR and proteinuria in Gujarati diabetics with mild-to-moderate nephropathy. J Family Med Prim Care. 2019;8:2965-70.

17 Samargandy S, Matthews KA, Brooks MM, Barinas-Mitchell E, Magnani JW, Janssen I. Arterial stiffness accelerates within 1 year of the final menstrual period: the SWAN heart study. Arterioscler Thromb Vasc Biol. 2020 Apr;40(4):1001-8. ATVBAHA-119.

18 Solanki JD, Makwana AH, Mehta HB, Gokhale PA, Shah CJ. Body composition in type 2 diabetes: change in quality and not just quantity that matters. Int J Prev Med. 2015;6: 122.

19 MartinezAguirre-Betolaza A, Mujika I, Fryer SM, Corres P, Gorostegi-Anduaga I, Arratibel-Imaz I, et al. Effects of different aerobic exercise programs on cardiac autonomic modulation and hemodynamics in hypertension: data from EXERDIET-HTA randomized trial. J Hum Hypertens. 2020;34:709-18.

20 Cicero AF, Rosticci M, Fogacci F, Grandi E, D’Addato S, Borghi C; Brisighella Heart Study Group. High serum uric acid is associated to poorly controlled blood pressure and higher arterial stiffness in hypertensive subjects. Eur J Intern Med. 2017;37:38-42.

21 Gordin D, Saraheimo M, Tuomikangas J, Soro-Paavonen A, Forsblom C, Paavonen K, et al. Influence of postprandial hyperglycemic conditions on arterial stiffness in patients with type 2 diabetes. J Clin Endocrinol Metab. 2016;101(3):1134-43.

22 Chang S, Kim J, Sohn T, Son H, Lee J. Effects of glucose control on arterial stiffness in patients with type 2 diabetes mellitus and hypertension: an observational study. J Int Med Res. 2018;46(1):284-92.

23 Solanki JD, Basida SD, Mehta HB, Panjwani SJ, Gadhavi BP, Patel P. Impact of disease control and co-existing risk factors on heart rate variability in Gujarati type 2 diabetics: an observational study. J Family Med Prim Care. 2016;5(2):393-8

24 Climie RE, van Sloten TT, Bruno RM, Taddei S, Empana JP, Stehouwer CDA, et al. Macrovasculature and microvasculature at the crossroads between type 2 diabetes mellitus and hypertension. Hypertension. 2019;73(6): 1138-49.

25 Solanki JD, Mehta HB, Shah CJ. Pulse wave analyzed cardiovascular parameters in young first degree relatives of type 2 diabetics- a cross-sectional study. Indian Heart J. 2018; 70(3):341-5.

26 Solanki JD, Mehta HB, Shah CJ. Pulse wave analyzed cardiovascular parameters in young first degree relatives of hypertensives. J Res Med Sci. 2018;23:72. 